\title{
Strong Near-Field Coupling of Plasmonic Resonators Embedded in Si Nanowires
}

Dmitriy S. Boyuk, Li-Wei Chou, and Michael A. Filler*

School of Chemical \& Biomolecular Engineering, Georgia Institute of Technology, Atlanta, Georgia, 30332,

United States

*E-mail: $\underline{\text { mfiller@gatech.edu }}$ 


\section{Isotropic Si Dielectric Environments:}

Figure S2a reveals that two LSPRs exist for coupled Si resonators embedded in isotropic Si. The field enhancement maps shown in the insets to Figures S2b and S2c confirms that both modes are dipolar. To determine a $\tau$ value for each mode, the frequency of each mode can be extracted via standard fitting procedures as a function of resonator spacing. As seen in Figure S2c and expected for coupled dipolar resonators, the stronger, low energy dipolar mode undergoes a monotonic redshift as resonator spacing is reduced. The $\tau$ value extracted for this mode is 0.4 , as shown in Figure 2, and is consistent with prior studies of isotropic dielectric environments. However, Figure S2b shows that the weaker, high energy dipolar mode first redshifts and then blueshifts as resonator spacing decreases. We attribute this complex behavior to a convolution of near-field coupling and energy sharing with the low energy mode, which is energetically similar. Unfortunately, this behavior precludes a straightforward determination of $\tau$ for this mode.

To better understand the coupling of resonators in isotropic Si environments, Figure S3 shows the calculated spectral responses of isolated resonators for several aspect ratios. An aspect ratio of 0.8 , which was used in experiment, yields modes of similar absorption efficiency. For this situation, one would expect the largest amount of energy sharing, which likely prevents a monotonic redshift as resonator spacing is reduced (vide supra). However, mode absorption efficiencies become increasingly distinct as aspect ratio is either increased or decreased from 0.8. As an example, shown in Figure S4, we calculated the spectral response for coupled resonators with an aspect ratio of 0.2 as a function of resonator spacing. Figure S5 shows the spectral response for each mode, as extracted via peak fitting. Here, as expected for simple dipolar coupling, both modes monotonically redshift as resonator spacing decreases. 

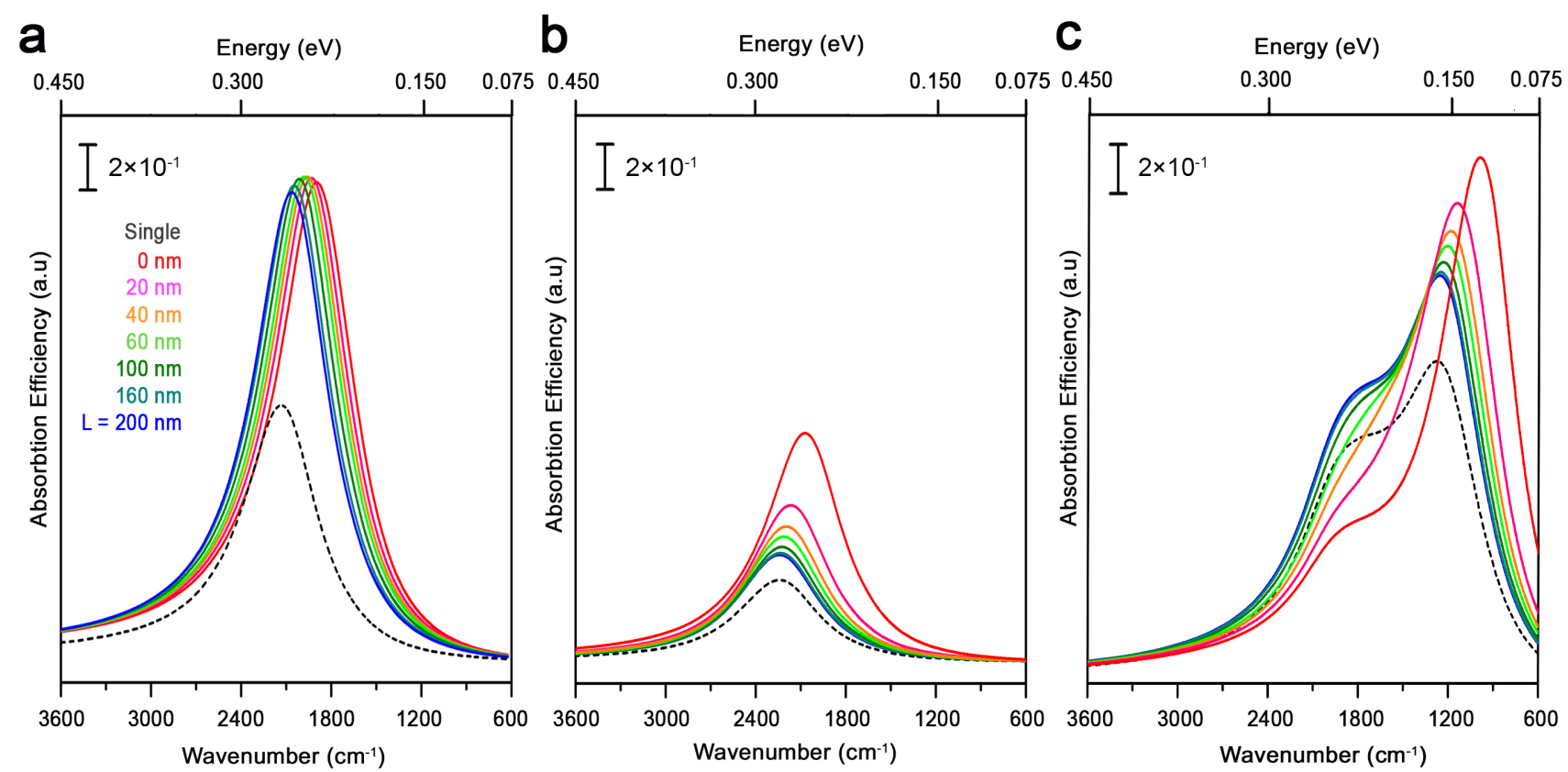

Figure S1. Calculated absorption efficiency spectra for coupled Si resonators as a function of resonator separation in (a) Si nanowire, (b) isotropic vacuum, and (c) isotropic Si dielectric environments. Resonator aspect ratios and carrier densities are all 0.8 and $2.7 \times 10^{20} \mathrm{~cm}^{-3}$, respectively. Calculated absorption efficiencies for isolated resonators with the same geometry and carrier density are included in each plot for comparison (black dotted curves). To match the dimensions of nanowires obtained experimentally, calculations are performed for cylindrical nanowires with a diameter and length of 130 and $950 \mathrm{~nm}$, respectively. 

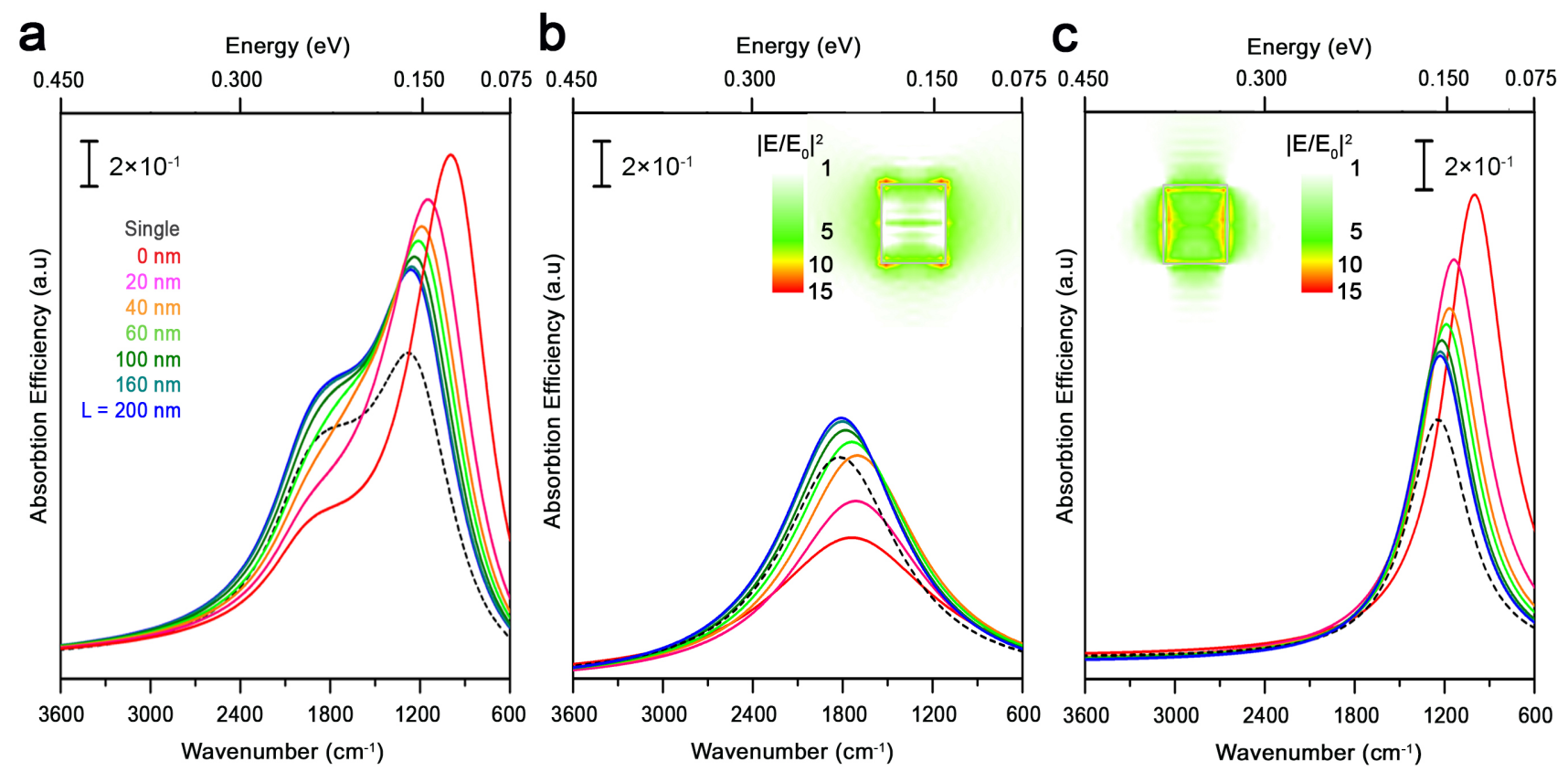

Figure S2. (a) Calculated absorption efficiency spectra for coupled Si resonators as a function of resonator separation in isotropic $\mathrm{Si}$ (same plot as Figure S1c). Resonator aspect ratio and carrier density are 0.8 and $2.7 \times$ $10^{20} \mathrm{~cm}^{-3}$, respectively. The (b) higher and (c) lower energy absorption modes extracted by peak fitting the data in (a) with two Lorentzians. The spectra in (c) are the basis of the plasmon ruler curve for coupled resonators embedded in isotropic Si shown in Figure 2. The insets in (b) and (c) show the calculated field enhancement maps for isolated resonators. 


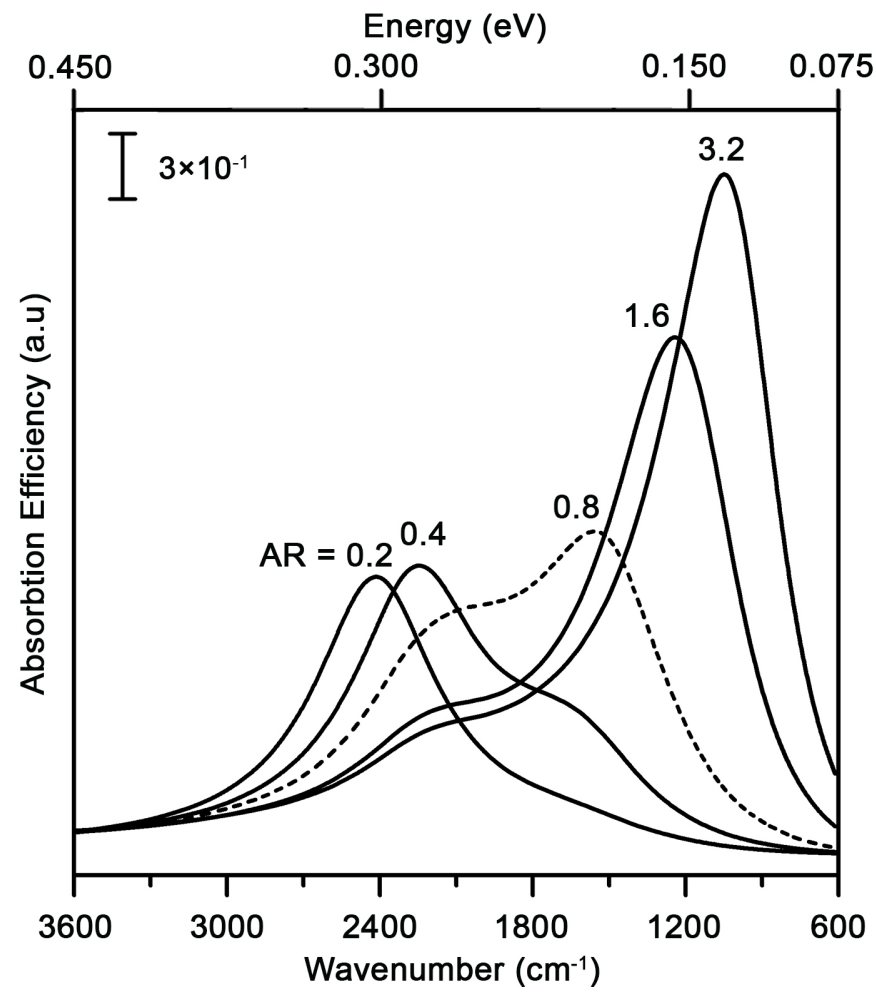

Figure S3. Absorption efficiency spectra calculated for single Si resonators embedded in isotropic Si. Each resonator has a carrier density of $2.7 \times 10^{20} \mathrm{~cm}^{-3}$ and aspect ratios between 0.2 and 3.2 . 


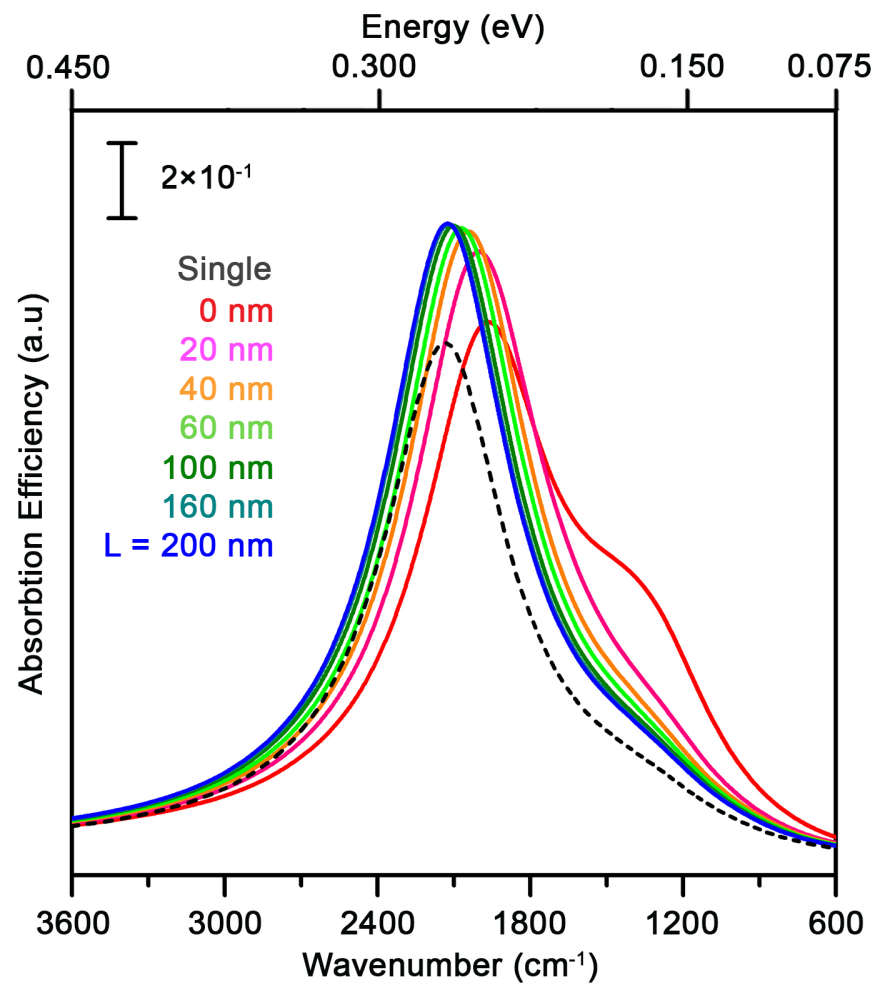

Figure S4. Absorption efficiency spectra calculated for coupled Si resonators embedded in isotropic Si as a function of separation distance. Resonator aspect ratio and carrier density are 0.2 and $2.7 \times 10^{20} \mathrm{~cm}^{-3}$, respectively. Calculated absorption efficiencies for isolated resonators with the same geometry and carrier density are included in each plot for comparison (black dotted curves). 


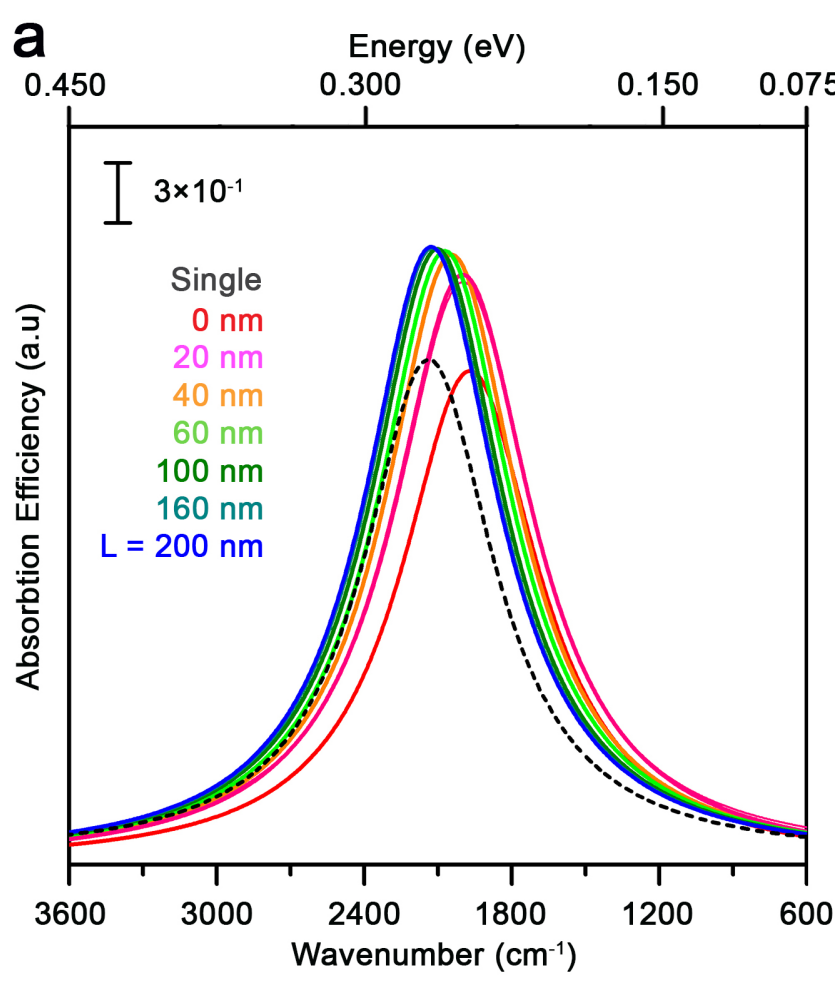

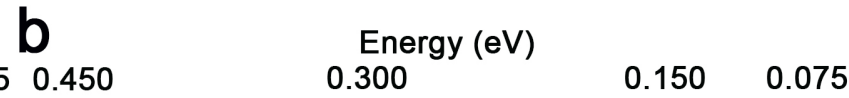

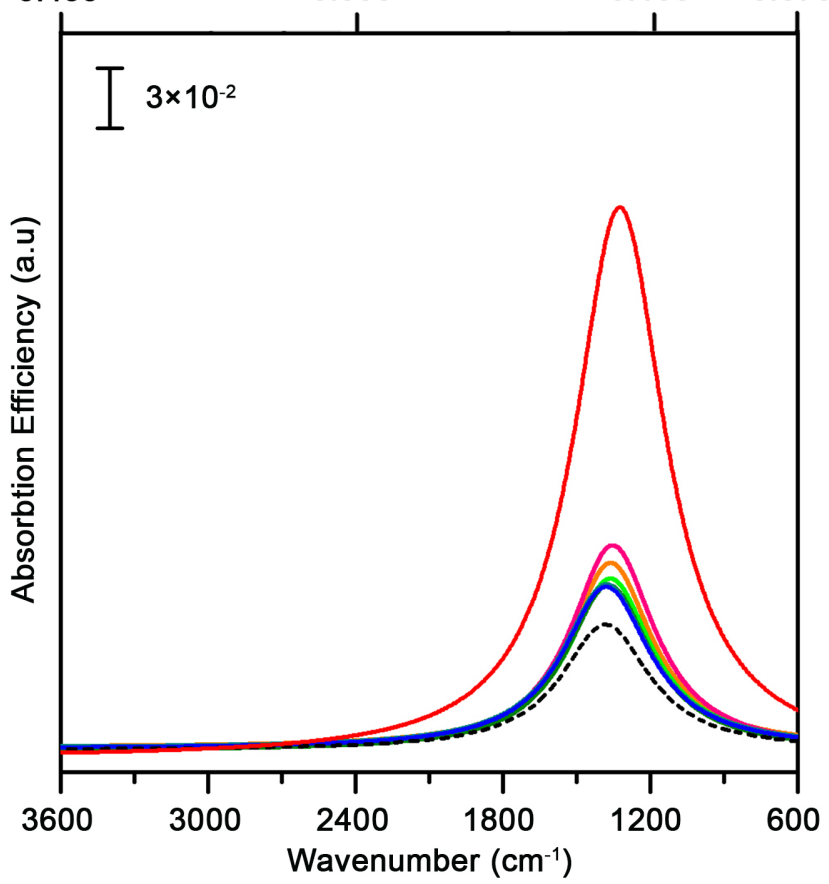

C
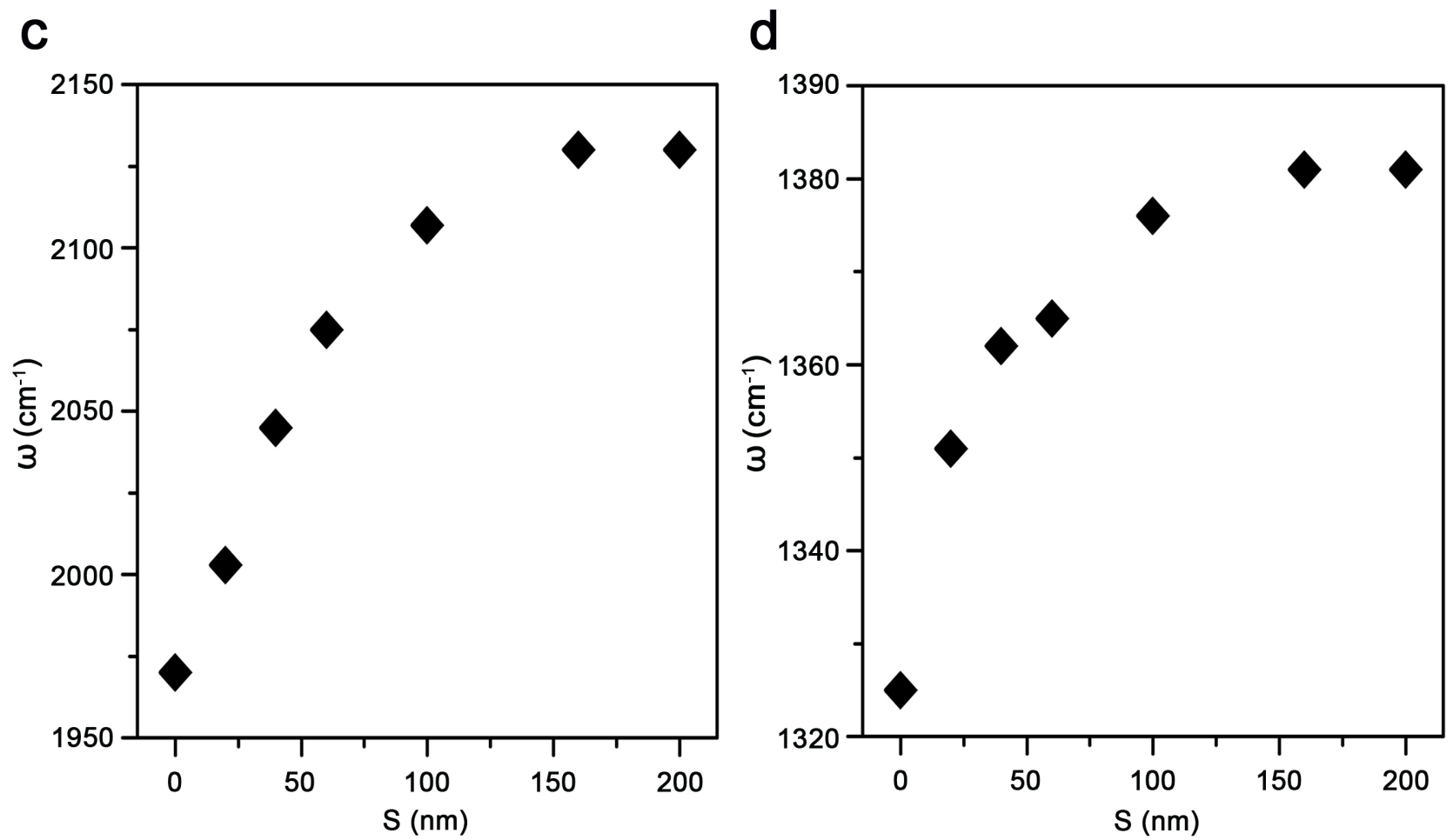

Figure S5. Absorption efficiency spectra for the (a) high and (b) low energy modes for coupled resonators embedded in isotropic $\mathrm{Si}$, as extracted from the data in Figure $\mathrm{S} 4$ with Lorentzian peak fitting. A monotonic redshift is observed for both modes, as quantified in (c) and (d). 


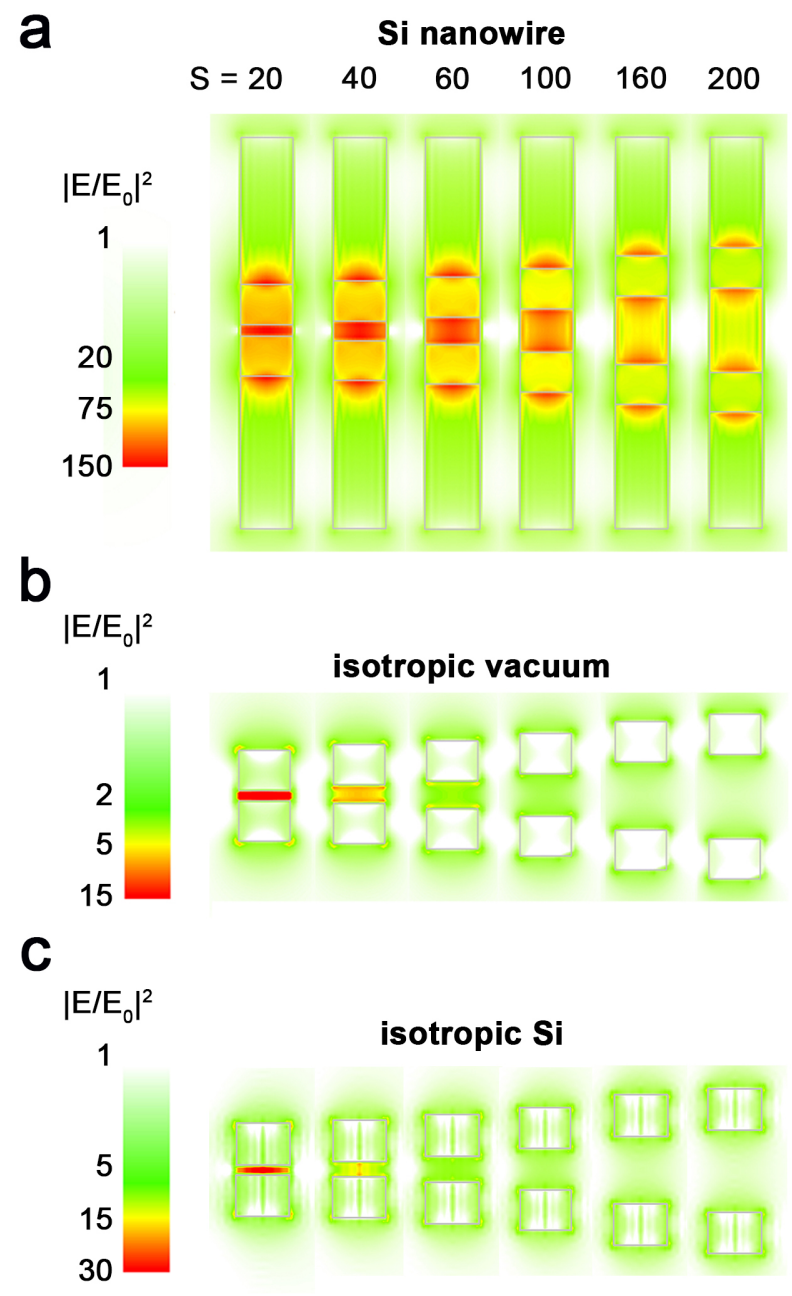

Figure S6. Calculated electric field enhancement maps for coupled Si resonators as a function of resonator separation in (a) Si nanowire, (b) isotropic vacuum, and (c) isotropic Si dielectric environments. Resonator aspect ratios and carrier densities are 0.8 and $2.7 \times 10^{20} \mathrm{~cm}^{-3}$, respectively. Each map is calculated at the frequency where absorption efficiency is at a maximum. Field enhancement magnitude is indicated by a red-togreen gradient. 


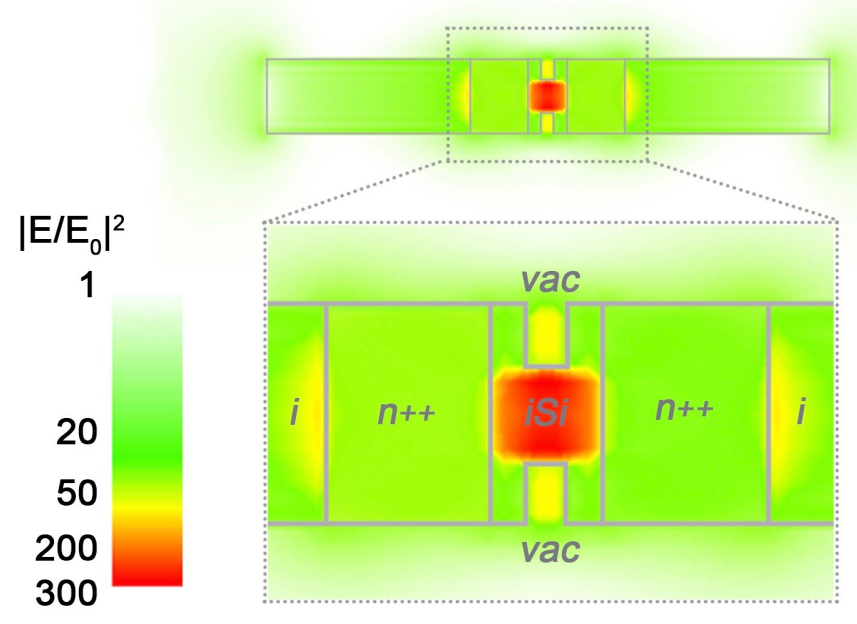

Figure S7. Calculated electric field enhancement map for coupled Si resonators separated by $60 \mathrm{~nm}$ with a 40 nm deep radial cut-out comprised of vacuum. Resonator aspect ratio and carrier density are 0.8 and $2.7 \times 10^{20}$ $\mathrm{cm}^{-3}$, respectively. Field enhancement magnitude is indicated by a red-to-green gradient. 


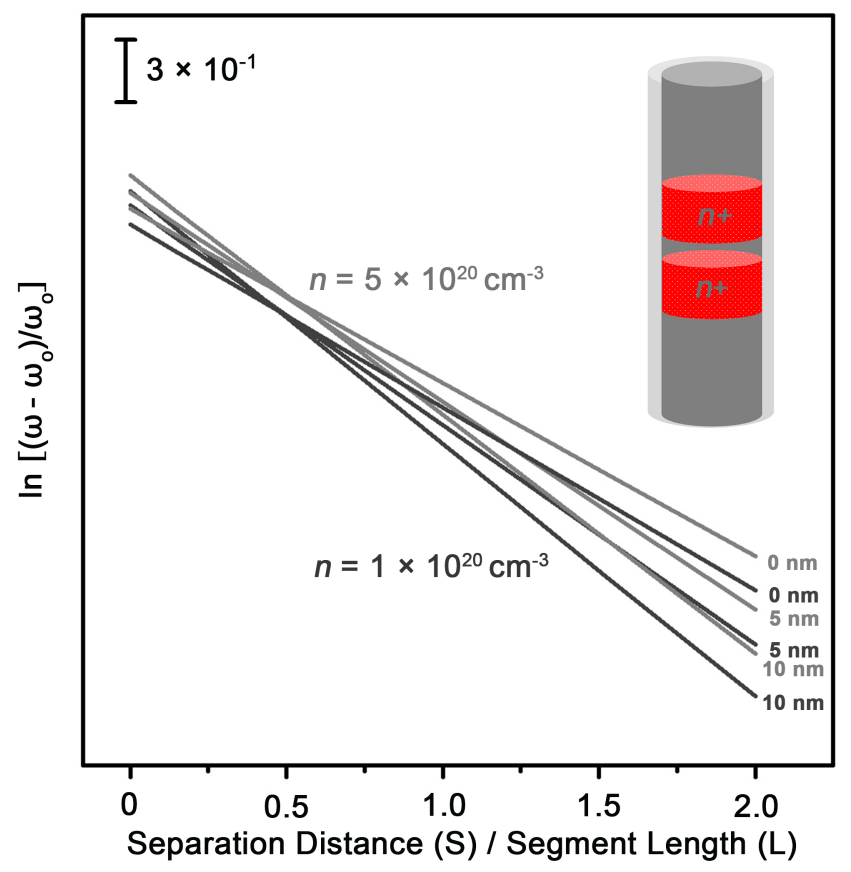

Figure S8. Effect of a uniform intrinsic Si shell on the decay length scaling factor. Calculated LSPR peak positions for nanowires containing coupled doped-Si segments fitted to a linearized form of equation 1 . Resonator carrier densities of $1 \times 10^{20} \mathrm{~cm}^{-3}$ (black) or $5 \times 10^{20} \mathrm{~cm}^{-3}$ (gray) and nanowire shell thicknesses of 0 , 5, and $10 \mathrm{~nm}$ are shown. Calculations are performed for cylindrical nanowires with a diameter and length of 130 and $950 \mathrm{~nm}$, respectively. A periodic grid spacing of $5 \mathrm{~nm}$ was used for these calculations. 